\title{
ON THE COMMUTATIVITY OF TORSION FREE RINGS
}

\section{EVAgelos Psomopoulos}

If $R$ is $(n(n-1) / 2)$-torsion free ring with 1 and satisfies the identity $(x y)^{n}=x^{n} y^{n}$, then $R$ is commutative provided that $n=4 k$.

A theorem of Bell [2] states that if a ring $R$ with identity 1 is $n$-torsion free and satisfies the two identities $(x y)^{n}=x^{n} y^{n}$ and $(x y)^{n+1}=x^{n+1} y^{n+1}$, then $R$ is commutative. In [1] Abu-Khuzam proved that if $R$ is $n(n-1)$-torsion free ring with 1 and satisfies the identity $(x y)^{n}=x^{n} y^{n}$, then $R$ is commutative. Recently Kobayashi [4] has stated the following conjecture: if $R$ is $(n(n-1) / 2)$-torsion free ring with 1 and satisfies the identity $(x y)^{n}=x^{n} y^{n}$, then $R$ is commutative provided that $n$ is even. Considering the ring

$$
R=\left\{\left(\begin{array}{lll}
a & b & c \\
0 & a^{2} & 0 \\
0 & 0 & a
\end{array}\right): a, b, c \in \mathrm{GF}(4)\right\}
$$

we see that, with $n=6, R$ is $(n(n-1) / 2)$-torsion free ring with 1 and satisfies the identity $(x y)^{n}=x^{n} y^{n}$. Note that $R$ is not commutative. Therefore, Kobayashi's conjecture is not true in general.

Received 9 April 1984.

Copyright Clearance Centre, Inc. Serial-fee code: 0004-9727/84 $\$ \mathrm{~A} 2.00+0.00$. 
However, we prove that if $n=4 k$, then the above conjecture is true. Namely, we prove the following

THEOREM. Let $n$ be a fixed positive integer. If $R$ is $n(2 n-1)-$ torsion free ming with 1 and satisfies the identity $(x y)^{2 n}=x^{2 n} y^{2 n}$, then $R$ is commutative provided that $n$ is even.

Throughout this note $R$ will be an associative ring with $1, Z(R)$ the center, $N(R)$ the set of all nilpotent elements and $C(R)$ the commutator ideal of $R$. As usual we write $[x, y]=x y-y x$.

We shall use freely the following well known results.

(I) If $[x,[x, y]]=0$, then $\left[x^{m}, y\right]=m x^{m-1}[x, y]$ for any positive integer $m$.

(II) If $x^{m}[x, y]=0$ for some positive integer $m$, then $[x, y]=0$.

We now proceed to prove our theorem.

Proof of the theorem. In hypothesis $(x y)^{2 n}=x^{2 n} y^{2 n}$ replace $x$ by $u^{-1} x$ and $y$ by $u$, where $u$ is an invertible element of $R$. Then

$$
u^{-2 n} x^{2 n} u^{2 n}=\left(u^{-1} x u\right)^{2 n}=u^{-1} x^{2 n} u
$$

which implies

(1) $\left[u^{2 n-1}, x^{2 n}\right]=0$, for all $x$ in $R$ and all invertible elements $u$ of $R$.

Let $a \in N(R)$; then there exists a positive integer $p$ such that

$$
\left[a^{k}, x^{2 n}\right]=0 \text {, for all } k \geq p, p \text { minimal. }
$$

Suppose $p>1$; then $1+a^{p-1}$ is invertible, and by (1) and (2) we obtain

$$
0=\left[\left(1+a^{p-1}\right)^{2 n-1}, x^{2 n}\right]=(2 n-1)\left[a^{p-1}, x^{2 n}\right]
$$

Since $R$ is (2n-1)-torsion free, we conclude that $\left[\alpha^{p-1}, x^{2 n}\right]=0$ which contradicts the minimality of $p$. Thus $p=1$ and (2) implies 


$$
\left[a, x^{2 n}\right]=0 \text {, for all } x \in R \text { and } a \in N(R) \text {. }
$$

Consider the subring $S=\left\langle x^{2 n}: x \in R\right\rangle$ of $R$ generated by all $2 n$th powers of elements of $R$. Then (3) implies $N(S) \subseteq Z(S)$, and by Herstein's theorem [3],

$$
C(S) \subseteq N(S) \subseteq Z(S)
$$

Since $(x y)^{2 n} x=x(y x)^{2 n}$ for all $x, y$ in $S$, we have

$$
\begin{aligned}
x^{2 n} y^{2 n} x & =x y^{2 n} x^{2 n}, \\
x\left[x^{2 n-1}, y^{2 n}\right] x & =0 .
\end{aligned}
$$

In view of (I) and (4) the last identity implies

$$
(2 n-1) x^{2 n}\left[x, y^{2 n}\right]=0 \text {, for all } x, y \text { in } S \text {. }
$$

But $S$ is $(2 n-1)$-torsion free, thus $x^{2 n}\left[x, y^{2 n}\right]=0$ which, in view of (II), implies

$$
\left[x, y^{2 n}\right]=0 \text {, for all } x, y \text { in } S \text {. }
$$

Applying (I), in view of (4), to the last identity we obtain

$$
2 n y^{2 n-1}[x, y]=0 \text {, for all } x, y \text { in } s
$$

Since $S$ is $n$-torsion free and $n$ is even, we conclude that

$y^{2 n-1}[x, y]=0$ which together with (II) implies

$$
[x, y]=0 \text {, for all } x, y \text { in } S \text {. }
$$

Therefore

$$
x^{2 n} y^{2 n}=y^{2 n} x^{2 n} \text {, for all } x, y \text { in } R
$$

Then

$$
x^{2 n+1} y^{2 n+1}=x\left(x^{2 n} y^{2 n}\right) y=x\left(y^{2 n} x^{2 n}\right) y=x(y x)^{2 n} y=(x y)^{2 n} x y=(x y)^{2 n+1}
$$

that is,

$$
(x y)^{2 n+1}=x^{2 n+1} y^{2 n+1} \text {, for all } x, y \text { in } R \text {. }
$$

Furthermore, 


$$
(x y)^{2 n}=x^{2 n} y^{2 n}=y^{2 n} x^{2 n}=(y x)^{2 n} .
$$

Since $(x y)^{2 n} x=x(y x)^{2 n}=x(x y)^{2 n}$, we have

$$
0=\left[x,(x y)^{2 n}\right]=\left[x, x^{2 n} y^{2 n}\right]=x^{2 n}\left[x, y^{2 n}\right] .
$$

Combining (II) and the last identity we obtain

$$
\left[x, y^{2 n}\right]=0 \text {, for all } x, y \text { in } R \text {. }
$$

Let $u$ be an invertible element of $R$. In (5) replace $x$ by $u^{-1} x$ and $y$ by $u$ to get

$$
u^{-1} x^{2 n+1} u=\left(u^{-1} x u\right)^{2 n+1}=u^{-2 n-1} x^{2 n+1} u^{2 n+1} \text {; }
$$

that is,

$$
\left[u^{2 n}, x^{2 n+1}\right]=0
$$

Now the same argument we used in (1) to obtain (3) works also in (7), since $R$ is $n$-torsion free and $n$ is even. Thus we can obtain

$$
\left[a, x^{2 n+1}\right]=0 \text {, for all } x \text { in } R \text { and } a \text { in } N(R) \text {. }
$$

Combining ( 3 ) and ( 8 ) we see that

$$
a \in Z(R) \text {, for all } a \text { in } N(R) \text {; }
$$

that is,

$$
N(R) \subseteq Z(R)
$$

Hence Herstein's theorem [3] implies

$$
C(R) \subseteq Z(R)
$$

Finally, combining (6), (9) and (I) we obtain

$$
0=\left[x, y^{2 n}\right]=2 n y^{2 n-1}[x, y] \text {, for all } x, y \text { in } R \text {. }
$$

Since $R$ is $n$-torsion free and $n$ even, the last identity implies $y^{2 n-1}[x, y]=0$ which together with (II) yields $[x, y]=0$, for all $x, y$ in $R$.

This completes the proof of the theorem. 


\section{References}

[1] H. Abu-Khuzam, "A commutativity theorem for rings", Math. Japon. 25 (1980), 593-595.

[2] H.E. Bell, "On the power map and ring commutativity", Canad. Math. Buz2. 21 (1978), 399-404.

[3] I.N. Herstein, "Power maps in rings", Michigan Math. J. 8 (1961), $29-32$.

[4] Y. Kobayashi, "Some polynomial identities and commutativity of rings, I", Proc. 14 th Sympos. Ring Theory, 1981, 1-8 (Matsumoto, Japan, 1981).

Department of Mathematics,

University of Thessaloniki,

Thessaloniki,

Greece. 